\title{
Electrophoretic manipulation of multiple-emulsion droplets
}

\author{
Andreas M. SCHOELER ${ }^{1,}{ }^{*}$, Dimitris N. JOSEPHIDES ${ }^{1}$, Ankur S. CHAURASIA ${ }^{1}$, Shahriar SAJJADI ${ }^{1}$, Patrick
}

$\underline{\text { MESQUIDA }^{1}}$

*Corresponding author: Tel.: ++44 (0)207 848 2902; Email: andreas.schoeler@kcl.ac.uk 1: Department of Physics, Kings College London, UK

\begin{abstract}
In this report the electrophoretic manipulation of structured oil-water-oil $(\mathrm{O} / \mathrm{W}) / \mathrm{O}$ core-shell droplets is presented. Water shells have been created that allow the electrophoretic manipulation of oil droplets in an oil environment. It was found that the inner droplet regardless of size and composition does not affect the electrophoretic mobility of the outer water shell, neither before nor after contact with a biased electrode. This method can be used for the manipulation of oil droplets in a continuous oil phase or for the transportation of microbial cells that would otherwise be killed at low electric field strengths.
\end{abstract}

Keywords: Electrophoresis, Micro Flow, Emulsion, Carrier Mobility

\section{Introduction}

Double emulsions, water-in-oil-in-water $((\mathrm{W} / \mathrm{O}) / \mathrm{W})$ and oil-in-water-in-oil $((\mathrm{O} / \mathrm{W}) / \mathrm{O})$, are an attractive tool in biomedical engineering as they offer liposome delivery vehicles (Shum et al., 2008), microcapsules (Chen et al., 2012), controlled content release (Abbaspourrad et al., 2013) or living cell encapsulation (Martinez et al., 2012).

Often electrostatic fields are used to manipulate droplets and information about the electrophoretic behaviour of charged water droplets has become more important, because repulsion or attraction between droplets can affect the performance of microfluidic devices (Millman et al., 2005). For example Im et al. (Im et al., 2011b) recently performed a feasibility study to test the influence of an electric field on living cells and did not observe any noticeable change in viability and proliferation of living cells, indicating that electrophoresis is a safe and feasible method for manipulating living biological systems. Cell manipulation often requires sterile techniques, for example laminar flow cabinets that prevent the contamination of biological samples or other kinds of particle sensitive devices. Electrophoresis offers a new technique for high precision manipulation of droplets and could replace such large bench designs with smaller lab-on-a-chip devices.
Choi et al. recently presented a droplet manipulation technique, whereby a water droplet is given a charge from an electrode and is then driven by electric fields (Choi et al., 2012). Apart from carrying a native charge, water-in-oil droplets can also be deliberately charged through direct contact with a voltagebiased electrode (Khayari et al., 2003). The great advantage it offers is the degree of control with respect to sign and magnitude of the charge. However some microbial cells, such as yeast, are killed at low electric field strengths (Grahl and Märkl, 1996; Sale and Hamilton, 1967; Van Loey et al., 2001) and could benefit by being encapsulated within a water droplet.

In order to investigate the electrophoretic mobility of complex microdroplets, we performed velocity measurements of large droplets, that is, droplets greater than $100 \mu \mathrm{m}$, by video microscopy. We employed a simple, electrophoretic set-up to investigate individual $(\mathrm{O} / \mathrm{W}) / \mathrm{O}$ droplets injected by glass microcapillaries and actuated by electrostatic fields between two closely spaced, biased electrodes.

\section{Experimental}

\subsection{Apparatus}

All micro-electrophoretic experiments were performed using the same transparent, 
electrophoretic cell and experimental method as described in (Schoeler et al., 2013) (Fig.1a). Individual oil-in-water droplets (deionized water, density $\rho_{\text {water }}=1000 \mathrm{kgm}^{-3}$, Millipore Direct-Q 3, Ultrapure Water Systems, Watford, UK) were injected into the continuous silicone oil phase (100 cSt silicone oil $\left(\mathrm{C}_{2} \mathrm{H}_{6} \mathrm{OSi}\right)_{\mathrm{n}}$, dynamic viscosity $\eta_{\text {silicone }}=0.106$ Pas, density $\rho_{\text {silicone }}=965 \mathrm{kgm}^{-3}$ electrical resistivity $\delta_{\text {silicone }}=10^{15} \Omega \mathrm{cm}$, dielectric permittivity $\varepsilon_{\text {silicone }}=2.43 \times 10^{-11} \mathrm{~F} / \mathrm{m}$, CAS-number $=63148-62-9, \quad$ Sigma-Aldrich, Dorset, UK) using a concentric, glass microcapillary channel system (outer channel diameter $=320 \mu \mathrm{m}, \quad$ inner channel diameter $=70 \mu \mathrm{m}$ ). Once injected, the coreshell droplets sink to the bottom of the cuvette with a gravitational terminal velocity, $v_{g}$. When a droplet reaches the middle between the $\mathrm{Cu}$ electrodes, the voltage, $U$, is applied, creating an electric field, $E$, that causes the droplet carrying an effective charge, $Q$, to be displaced with a horizontal velocity component, $v_{e l}$ (Schoeler et al., 2013). The velocity of the droplets was measured in the middle of the cuvette to minimize any sidewall effects and where the terminal velocity, $v_{e l}$, has been reached (Fig.1a). The water shell radius was kept constant $\left(R_{\text {shell }}=800 \mu \mathrm{m}\right)$, whilst the radius of the oil core droplet was variable $\left(R_{\text {core }}=0-750 \mu \mathrm{m}\right)$. All experiments were conducted at room temperature $\left(21^{\circ} \mathrm{C}\right)$. (a)

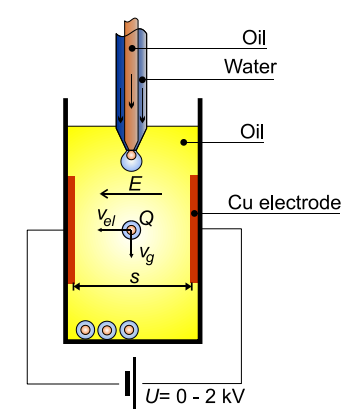

(c)

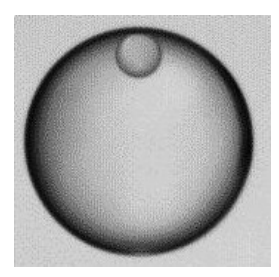

Ratio $=0.19$

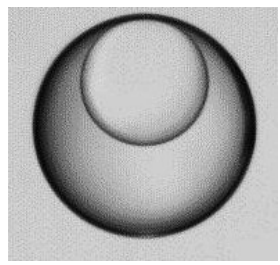

0.35 (b)

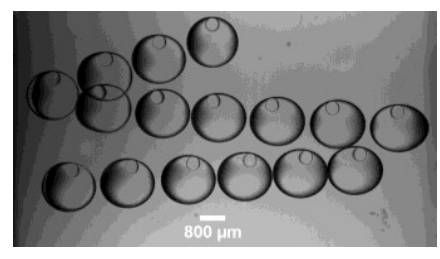

$800 \mu \mathrm{m}$
Figure 1 (a) Vertical set-up for electrophoretic measurements. (b) Back-and-forth motion of the $\mathrm{O} / \mathrm{W} / \mathrm{O}$ droplet between the two electrodes while continuing to slowly fall due to gravity. Both oil phases are silicone oil. (c) Images of droplets with different radius ratios using $2.30 \mathrm{~g} / 1 \mathrm{SDS}$

\section{Results}

\subsection{General behaviour of $(\mathrm{O} / \mathrm{W}) / \mathrm{O}$ droplets}

In the absence of surfactant it was not possible to create stable $(\mathrm{O} / \mathrm{W}) / \mathrm{O}$ droplets at $R_{\text {core }}>500 \mu \mathrm{m}$, as the oil core would merge with the continuous oil phase. For $(\mathrm{O} / \mathrm{W}) / \mathrm{O}$ droplets with smaller oil core sizes it was observed that, as soon as the electric field was applied, all droplets initially travelled towards the negative electrode, thereby showing that pure core-shell droplets have an initial, positive electrophoretic mobility before making contact with an electrode. Near the electrode the droplets elongated slightly towards the electrode, forming a small tip, which eventually touched the electrode (Fig. 1b). Upon touching the electrode for a fraction of a second, the droplets were repelled and travelled towards the other electrode (Fig. 1b), where the process repeated itself in the opposite direction leading to a back-andforth motion between the two electrodes while continuing to slowly fall due to gravity.

The magnitudes of the droplet velocity are 
very different before and after the first contact with an electrode, i.e. for a given droplet radius ratio, the velocity after first contact is approximately $10 \times$ greater than the initial velocity (Fig. 3). For example, for a droplet radius ratio $\left(R_{\text {core }} / R_{\text {shell }}\right)$ of 0.40 , the initial velocity is typically of the order of $10^{-3} \mathrm{~ms}^{-1}$, whereas the velocity after first contact is typically of the order of $10^{-2} \mathrm{~ms}^{-1}$. This general behaviour of an initial charge followed by active charging has been observed by other groups (Bailes et al., 2000; Hase et al., 2006; Im et al., 2012; Im et al., 2011a; Jalaal et al., 2010; Jung et al., 2008), although so far no other group has attempted experiments with complex-multiple droplets. As the core-shell droplet was detached from the microcapillary the inner oil droplet was horizontally centred inside the water shell (Fig. 1b). It was observed that as soon as the electric field was applied the relative motion of the oil core appeared to be in the opposite direction compared to the outer water shell (Fig. 1b).

Adding $2.3 \mathrm{~g} / 1$ of the anionic surfactant SDS meant that all droplets initially travelled towards the positive electrode, thereby showing that in the presence of SDS core-shell droplets have an initial, negative effective electrophoretic mobility before making contact with an electrode. The SDS core-shell droplets then followed the same motion as pure droplets, i.e. a back-and-forth motion between the two electrodes while continuing to slowly fall due to gravity.

Higher deformation of the water droplet compared to the previous case was observed and can be explained by the lowered interfacial tension. The addition of surfactant also allowed the core-shell droplets to become more stable (i.e. the oil core would no longer exit from the outer water shell) allowing greater core-shell size control (Fig. 1c) and larger core/shell ratios to be created (Fig. 2 and 3). Unlike the outer water shell no elongation or other droplet deformation could be observed for the oil core (Fig. 4).

\subsection{Influence of the oil core size of $(\mathrm{O} / \mathrm{W}) / \mathrm{O}$ droplets on the electrophoretic mobility}

Initial experiments were conducted using a simple oil-water-oil system (100 cSt Silicone Oil, no surfactant). Due to the instability (i.e. exit of oil core from outer water shell) with larger core sizes, a droplet radius ratio greater than 0.6 could not be achieved. In order to measure the droplet's electrophoretic velocity, $v_{e l}$, high-speed images $(100 \mathrm{fps}, 20 \mu \mathrm{m} / \mathrm{pixel})$ of individual, moving droplets were recorded and analysed using an adapted version of the Circular Hough Transform Function in Matlab, where droplets were tracked over time. Figure 2 shows that the initial droplet velocity of a droplet without a core $\left(R_{\text {core }} / R_{\text {shell }}=0\right)$ is much greater than the velocity of a core-shell droplet. On the other hand the droplet velocity appears to be of similar magnitude after the shell has made contact with a biased negative electrode regardless of droplet core/shell radius ratio (Fig. 3), suggesting that doubleemulsion $(\mathrm{O} / \mathrm{W}) / \mathrm{O}$ droplets have essentially the same, or not significantly different, electrophoretic mobility as simple W/O droplets under otherwise identical conditions (oil type, surfactant, contact with biased electrode, etc.) (Schoeler et al., 2013).

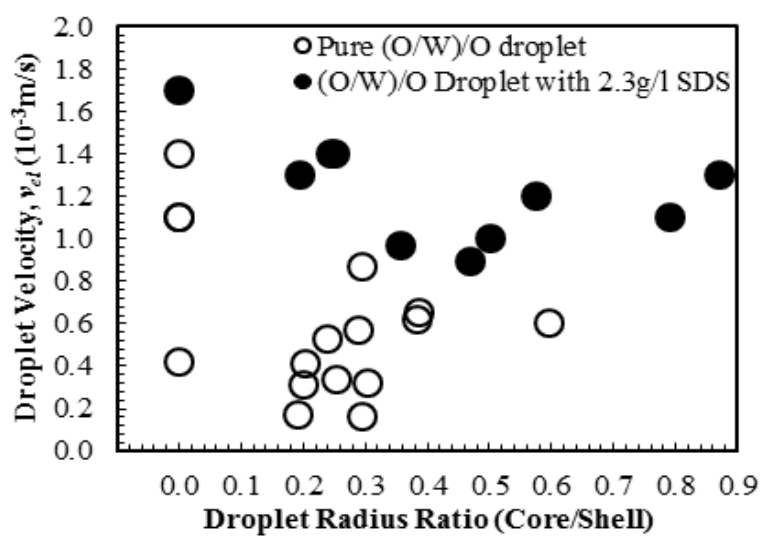

Figure 2 Magnitudes of electrophoretic droplet velocity, $v_{e l}$, of an $(\mathrm{O} / \mathrm{W}) / \mathrm{O}$ droplet in dependence of oil core size, where $R_{\text {shell }}=$ constant $=800 \mu \mathrm{m}$ and $R_{\text {core }}=0-750 \mu \mathrm{m} \quad$ before contact with an electrode, with and without surfactant

With the addition of the anionic surfactant SDS $(2.30 \mathrm{~g} / 1)$ it was possible to create more stable core-shell droplets and as a result a droplet radius ratio of up to 0.93 was achieved (Fig. 1c). It is well established that the addition of the anionic surfactant SDS causes the surface charge of droplets to be negative $(\mathrm{Gu}$ and $\mathrm{Li}, 1998)$, which is why the initial surface charge of our outer water shell with $2.3 \mathrm{~g} / 1$ 
SDS was altered from positive to negative. Figure 2 shows that the magnitude of the droplet velocity decreases with the addition of a core. From Figure 3 one can see that as soon as the droplet makes contact with an electrode (in this case a biased positive electrode), the magnitude of the droplet velocity remains constant regardless of core size.

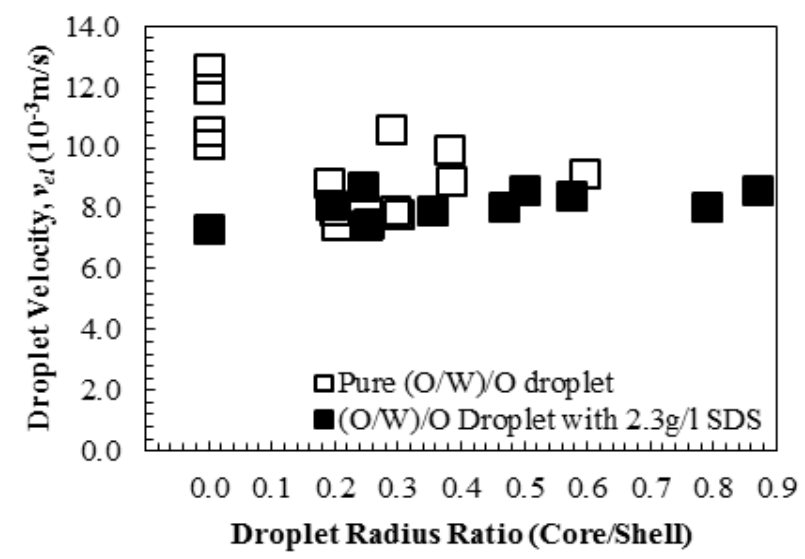

Figure 3 Magnitudes of electrophoretic droplet velocity, $v_{e l}$, of an $(\mathrm{O} / \mathrm{W}) / \mathrm{O}$ droplet in dependence of oil core size, where $R_{\text {shell }}=$ constant $=800 \mu \mathrm{m}$ and $R_{\text {core }}=0-750 \mu \mathrm{m}$ after contact with an electrode, with and without surfactant

From this observation, one can conclude that any charge of the oil core is probably screened by the outer water shell. The implications for practical applications are, thus, that complex droplets can be manipulated, and will react, in very much the same way as simple droplets. Furthermore, small, charged oil cores can be seen as electrostatically equivalent to globular proteins or cells encapsulated in the water droplets. This means that it is unlikely that the addition of charged particles or cells into water microdroplets would greatly affect any electrostatic manipulation procedures in microfluidic devices, which is compatible with previous results, where we did not see any significant influence of electrolyte ions on the electrophoretic mobility of W/O droplets (Schoeler et al., 2013).

\subsection{Droplet deformation}

Jung et al. (Jung et al., 2008) reported that a single deionised water droplet, of radius $R_{\min }$, deforms into a slightly elongated shape (to a radius $R_{\max }$ ) as it approaches an electrode. It is argued that the electric field becomes stronger in the gap between the droplet's surface and the electrode and the droplet surface charges become concentrated, causing the droplet to deform into an elongated shape with a sharper tip. Further reducing the interfacial tension between the droplet and the continuous phase by adding surfactant increases the droplet deformability and causes the droplet to elongate more as the electric field is applied. As velocities of $v_{g}$ and $v_{e l}$ before first contact with an electrode are of comparable magnitudes and having observed no droplet deformation in free fall, we are certain that droplet deformation is due the electrical field applied rather than shear. We observed that the droplet further elongates shortly before, at contact, and shortly after contact with an electrode and have found that the oil core droplet does not deform at all - neither before nor after contact with an electrode (Fig. 4).

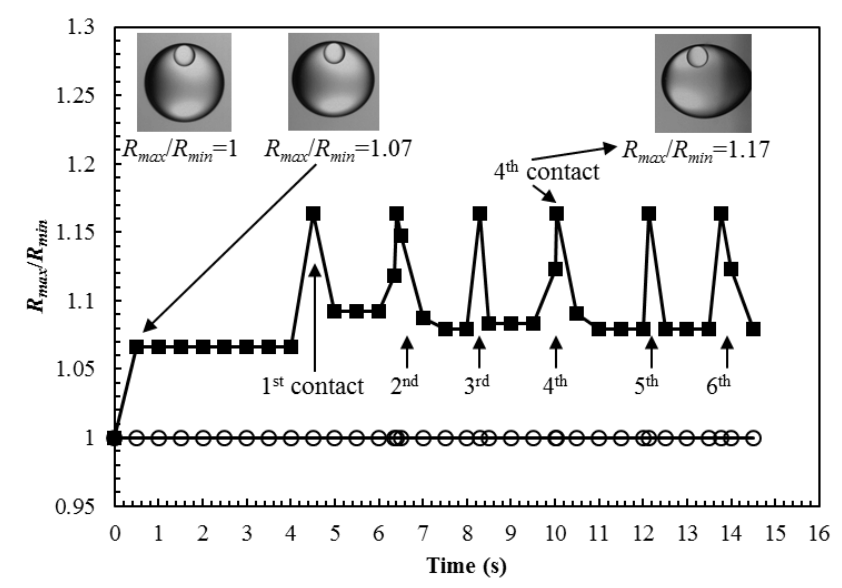

Figure 4 Droplet deformation of the outer water droplet with of $2.30 \mathrm{~g} / 1 \mathrm{SDS}$, shell drop deformation $(\boldsymbol{\square})$ and core drop deformation $(\mathrm{O})$. First contact is with a biased positive electrode. The straight lines between data points are only guidelines to indicate the sequence of measurements..

\subsection{Deflection of the oil core against the direction of motion in electrostatic fields}

As several other research groups before, we observed that water droplets clearly undergo discharging and re-charging with opposite polarity when making contact with a biased electrode (Hase et al., 2006; Im et al., 2012; Im et al., 2011a; Jalaal et al., 2010; Jung et al., 2008; Lee et al., 2012; Takinoue et al., 2010; Vajdi Hokmabad et al., 2012). We also observed that the initially horizontally centred oil core appears to be moving in the opposite 
direction of the water shell, when an electric field is applied, regardless of field direction, illustrated in Figure 1b.

To investigate this further, we preformed additional experiments for $(\mathrm{O} / \mathrm{W}) / \mathrm{O}$ droplets with $2.30 \mathrm{~g} / 1 \mathrm{SDS}$ (with varying core sizes), where one electrode was insulated using one layer of Parafilm M (Sigma-Aldrich, Dorset, $\mathrm{UK})$. The initially negatively charged $(\mathrm{O} / \mathrm{W}) / \mathrm{O}$ droplet would travel towards the positive electrode where upon touching the electrode for a fraction of a second, the $(\mathrm{O} / \mathrm{W}) / \mathrm{O}$ droplet was repelled and travelled towards the other electrode. Upon touching the insulated electrode the $(\mathrm{O} / \mathrm{W}) / \mathrm{O}$ droplet would stick to the Parafilm, which inhibited charge transfer. When the direction of the electric field was alternated, it was observed that the oil core droplets did not move (Fig. 5a and b), which is contrary to what has been observed above and is illustrated in Figure $5 \mathrm{c}$ and $\mathrm{d}$. This indicates that the motion of the inner droplet is most likely due to the internal circulation of the water droplet rather than the oil droplet carrying an opposite surface charge to the water droplet. (a)

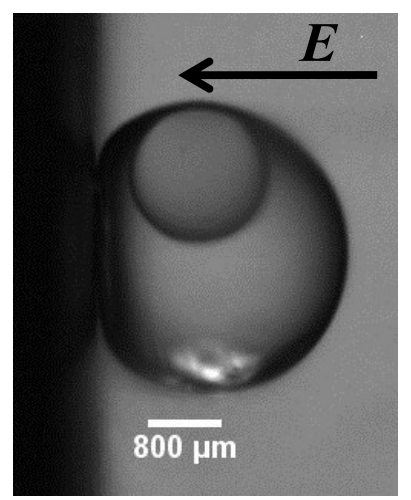

(c)

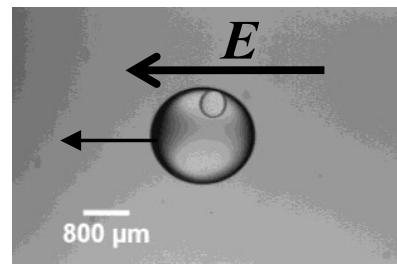

(b)

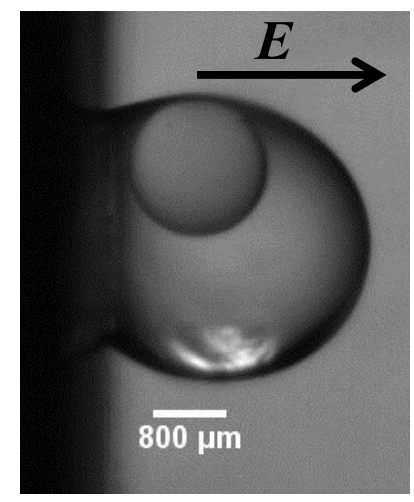

(d)

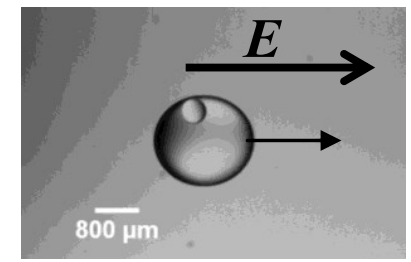

Figure 5 Difference in oil core position within the outer water shell between a moving core-shell and a stationary core-shell droplet. a and b) $(\mathrm{O} / \mathrm{W}) / \mathrm{O}$ droplet (with $2.30 \mathrm{~g} / \mathrm{l} \mathrm{SDS})$ attached to an insulated electrode (left). a) negative electrode, b) positive electrode. $\mathrm{c}$ and d) Electrophoretic motion of an $(\mathrm{O} / \mathrm{W}) / \mathrm{O}$ droplet (with $2.30 \mathrm{~g} / \mathrm{l} \mathrm{SDS}$ ) away from an electrode, where the arrows indicate the direction of travel. c) oil core deflected to the right with respect to the centre of the outer water shell, against the direction of motion, d) oil core deflected to the left with respect to the centre of the outer water shell, against the direction of motion.

\section{Discussion}

At first glance this could lead one to believe that the reduction in the electrophoretic mobility of a complex droplet is due to the fact that an oil core dispersed in an outer water shell carries a surface charge that is opposite to that of the shell. Thus if an electric field is applied the initially positively charged outer water shell is attracted to the negative electrode, whilst the initially negatively charged oil core is attracted to the positive electrode. However this theory seems unlikely as the shell's electrophoretic mobility is unaffected by an increasing core size (Fig. 2 and 3 ) and the oil core does not deform in an electrical field (Fig. 4). One would also expect a reduction in electric field strength inside the water shell, due to the change in dielectric constant between the continuous oil phase and the water shell, which could shield the oil core. In this paper we have shown that the initial electrophoretic velocity, $v_{e l}$, of a water droplet is reduced when a silicone oil core droplet is introduced. We also observed that the magnitude of the velocity of the $(\mathrm{O} / \mathrm{W}) / \mathrm{O}$ droplet remains the same regardless of core size and that as soon as the water shell touches a biased electrode the magnitude of the velocity between a simple and a complex drop is the same. In an additional experiment an extra water core was added to create a $(\mathrm{W} / \mathrm{O} / \mathrm{W}) / \mathrm{O}$ droplet, but no increase in the initial electrophoretic mobility could be observed. If the internal interfaces were to carry a charge one would expect to see a higher surface charge, due to the addition of an extra water interface and thus a greater electrophoretic mobility of the $(\mathrm{W} / \mathrm{O} / \mathrm{W}) / \mathrm{O}$ droplet - however that was not the case. This would suggest a shielding effect of the outer water shell, meaning that the inner core droplets do not experience the same field strength as the outer water shell. This leads us to conclude that internal circulation of the droplets is a more likely explanation for the 
causes the core displacement and we would suggest that particle image velocimetry of this core-shell droplet behaviour should be employed to provide additional proof.

\section{Conclusion}

To summarise, this is a first report on electrophoretic manipulation of complex oil-in-water-in-oil (core-shell) drops. Thin water shells have been created that allow the electrophoretic manipulation of oil droplets in an oil environment. It was found that the inner droplet regardless of size and composition does not affect the electrophoretic motion caused by the application of an electric field neither before nor after the outer water shell has been in contact with an electrode. Furthermore it has been observed that the surface charge of the outer water shell can be altered through contact with an electrode and remains constant regardless of the oil core size. This is advantageous in a variety of applications. For example oil droplets of varying types and sizes can accurately be transported and manipulated at the same speed using a monodisperse water shell that can be either be thick or ultrathin. This technique can also be used for the manipulation of materials that would otherwise be damaged by an electrical field (Grahl and Märkl, 1996; Sale and Hamilton, 1967; Van Loey et al., 2001), such as enzymes, which could be manipulated using a complex $(\mathrm{W} / \mathrm{O} / \mathrm{W}) / \mathrm{O}$ droplet system.

\section{Acknowledgments}

The authors would like to thank the Engineering and Physical Sciences Research Council (EPSRC) for funding of this project through a DTA studentship (EPSRC 2009 DTA - Grant Ref: EP/P504961/1) and acknowledge the use made of Photron XLR Core Express, which was borrowed from the EPSRC Engineering Instrument Pool.

\section{References}

Abbaspourrad, A., Carroll, N.J., Kim, S.H., Weitz, D.A., 2013. Polymer Microcapsules with Programmable Active Release. J Am Chem Soc 135, 7744-7750.

Bailes, P.J., Lee, J.G.M., Parsons, A.R., 2000.
An experimental investigation into the motion of a single drop in a pulsed DC electric field. Chem. Eng. Res. Des. 78, 499-505.

Chen, P.W., Erb, R.M., Studart, A.R., 2012. Designer Polymer-Based Microcapsules Made Using Microfluidics. Langmuir 28, 144-152.

Choi, K., Im, M., Choi, J.M., Choi, Y.K., 2012. Droplet transportation using a pre-charging method for digital microfluidics. Microfluidics and Nanofluidics 12, 821-827.

Grahl, T., Märkl, H., 1996. Killing of microorganisms by pulsed electric fields. Appl Microbiol Biotechnol 45, 148-157.

Gu, Y.G., Li, D.Q., 1998. Electric charge on small silicone oil droplets dispersed in ionic surfactant solutions. Colloid Surface A 139, 213-225.

Hase, M., Watanabe, S.N., Yoshikawa, K., 2006. Rhythmic motion of a droplet under a dc electric field. Phys. Rev. E 74, 046301-046301 - 046301-046304.

Im, D.J., Ahn, M.M., Yoo, B.S., Moon, D., Lee, D.W., Kang, I.S., 2012. Discrete Electrostatic Charge Transfer by the Electrophoresis of a Charged Droplet in a Dielectric Liquid. Langmuir 28, 11656-11661. Im, D.J., Noh, J., Moon, D., Kang, I.S., 2011 a. Electrophoresis of a Charged Droplet in a Dielectric Liquid for Droplet Actuation. Anal Chem 83, 5168-5174.

Im, D.J., Noh, J., Yi, N.W., Park, J., Kang, I.S., 2011b. Influences of electric field on living cells in a charged water-in-oil droplet under electrophoretic actuation. Biomicrofluidics 5.

Jalaal, M., Khorshidi, B., Esmaeilzadeh, E., 2010. An experimental study on the motion, deformation and electrical charging of water drops falling in oil in the presence of high voltage DC electric field. Exp Therm Fluid Sci 34, 1498-1506.

Jung, Y.-M., Oh, H.-C., Kang, I.S., 2008. Electrical charging of a conducting water droplet in a dielectric fluid on the electrode surface. Journal of Colloid and Interface Science 322, 617-623.

Khayari, A., Perez, A.T., Garcia, F.J., Castellanos, A., 2003. Dynamics and deformation of a drop in a DC electric field. 2003 Annual Report Conference on Electrical Insulation and Dielectric Phenomena, 682-685. 
Lee, D.W., Im, D.J., Kang, I.S., 2012. Electrophoretic motion of a charged water droplet near an oil-air interface. Applied Physics Letters 100.

Martinez, C.J., Kim, J.W., Ye, C.W., Ortiz, I., Rowat, A.C., Marquez, M., Weitz, D., 2012. A Microfluidic Approach to Encapsulate Living Cells in Uniform Alginate Hydrogel Microparticles. Macromol Biosci 12, 946-951. Millman, J.R., Bhatt, K.H., Prevo, B.G., Velev, O.D., 2005. Anisotropic particle synthesis in dielectrophoretically controlled microdroplet reactors. Nat Mater 4, 98-102.

Sale, A.J.H., Hamilton, W.A., 1967. Effects of high electric fields on microorganisms: I. Killing of bacteria and yeasts. Biochimica et Biophysica Acta (BBA) - General Subjects 148, 781-788.

Schoeler, A.M., Josephides, D.N., Sajjadi, S., Lorenz, C.D., Mesquida, P., 2013. Charge of water droplets in non-polar oils. J Appl Phys 114, 144903.

Shum, H.C., Lee, D., Yoon, I., Kodger, T., Weitz, D.A., 2008. Double emulsion templated monodisperse phospholipid vesicles. Langmuir 24, 7651-7653.

Takinoue, M., Atsumi, Y., Yoshikawa, K., 2010. Rotary motion driven by a direct current electric field. Applied Physics Letters 96.

Vajdi Hokmabad, B., Sadri, B., Charan, M.R., Esmaeilzadeh, E., 2012. An experimental investigation on hydrodynamics of charged water droplets in dielectric liquid medium in the presence of electric field. Colloids and Surfaces A: Physicochemical and Engineering Aspects 401, 17-28.

Van Loey, A., Verachtert, B., Hendrickx, M., 2001. Effects of high electric field pulses on enzymes. Trends in Food Science \& Technology 12, 94-102. 